\title{
PROTECTIVE EFFECT OF GLYCYRRHIZC ACID AGAINST CARBON TETRACHLORIDE-INDUCED LIVER FIBROSIS IN RATS: ROLE OF INTEGRIN SUBUNIT $\beta$ LIKE 1 (ITG B L1)
}

\author{
Amal Ragab, Nasr Elsayed Nasr*, Khalid Kahilo \\ Biochemistry Department, Faculty of Veterinary Medicine Kafrelsheikh University \\ ${ }^{*}$ Corresponding author, E-mail: nasr_157@yahoo.com
}

\begin{abstract}
Glycyrrhizc acid (GA) is one of the herbal plants with a proved hepato-protective effect. The current study was carried out to estimate the hepato-protective effect of GA against liver fibrosis and to disclose its mode of action. Thirty two male albino rats were randomly distributed into 4 groups $(n=8)$, i.e., control group, $\mathrm{GA}$ group, $\mathrm{CCl}_{4}$ group and $\mathrm{CCl}_{4}+\mathrm{GA}$ group. Liver enzymes alanine aminotransferase (ALT), aspartate aminotransferase (AST), gamma-glutamyl transferase (GGT), liver histopathology, malondialdehyde (MDA), superoxide dismutase (SOD), catalase (CT) and gene expression of integrin subunit $\beta$ like 1 (ITGBL1) were analyzed. The obtained data revealed that GA remarkably protected $\mathrm{CCl}_{4}$-induced liver injury as reflected by reduced AST, ALT, GGT, and fibrosis compared with the $\mathrm{CCl}_{4}$-only group. Moreover, GA significantly reduced the levels of MDA, as well as increased the activity of SOD and CT. Consequently, GA prevents $\mathrm{CCl}_{4}$-induced fibrosis in rats. The prophylactic action of $\mathrm{GA}$ against liver fibrosis was mediated through its antioxidant and anti-inflammatory activities. Additionally, GA downregulated fibrogenic ITGBL1.
\end{abstract}

Key words: glycyrrhizc acid; ITGBL1; liver fibrosis; integrins

\section{Introduction}

Liver fibrotic diseases are generally resulted from chronic liver injury, leading to chronic inflammation and fibrosis with obliteration of the normal hepatic tissue construction and eventually loss of liver function (1). As a result of chronic liver diseases, cirrhosis is the sequelae of advanced liver fibrosis. Recently, it is reported that cirrhosis could be reversible in its early stages if the underlying liver diseases have been properly treated (2). However, advanced stages of cirrhosis are considered to be irreversible. Cirrhosis is believed to be the main source of a variety of serious complications, which lead to highly fatal systemic disorders (3). Therefore, cirrhosis the $8^{\text {th }}$ deadliest disease and is responsible for high percent of mortality worldwide. Worryingly, the prevalence of cirrhosis is increasing in numerous nations, including well developed ones (4). Chronic liver diseases such as alcoholic liver disease, $\mathrm{HCV}$, $\mathrm{HBV}$, haemochromatosis and non-alcoholic fatty liver disease (NAFLD) are the most frequent causes of cirrhosis worldwide. Despite their lower incidence rate, a wide range of other diseases can end with cirrhosis (5). Unfortunately, there is a shortage of an effective remedy for liver fibrosis to date. Therefore, there 
is an urgent requisite for potent anti-fibrotic remedies.

Fibrogenesis is a highly complicated process employing a variety of different cells, including hepatocytes, hepatic stellate cells (HSCs) and immunocytes. The key cellular elements in development of liver fibrosis is the activation of HSC and their trans-differentiation into myofibroblast-like cells (6). HSC activation is mainly initiated by growth factors and fibrogenic cytokines released by activated epithelial cells (including hepatocytes and cholangiocytes) and inflammatory cells, which among them the TGF $\beta 1$ is the key regulator (7).

Wide range genes take part in fibrogenesis through controlling the TGF $\beta$ signaling pathway, among which integrins have crucial regulatory role. Integrins are cellular receptors that consist of an $\alpha$ and a $\beta$ subunit and form at least 24 different dimers that mediate cell-cell and cell-ECM interactions (8). Moreover, integrins also response to ECM-induced extracellular changes during pathological processes, initiating cellular responses, which manipulate ECM alteration (9). It has previously showed that integrins manage pivotal roles in fibrogenesis (7). Throughout biliary fibrosis development, integrin $\alpha v \beta 6$ is highly upregulated in cholangiocytes and stimulate fibrogenesis through TGF $\beta$ 1 activation (10). Several genes and signaling pathways highly connected to fibrotic progression were discovered, among which integrin subunit $\beta$ like 1 (ITGBL1) was recognized as a key factor (11). The pathological pathway of ITGBL1 were clearly demonstrated by using in vitro experiments, which revealed that ITGBL1 encourages HSC activation and the subsequent liver fibrosis by upregulating TGF $\beta 1$. These observations secure essential base for further research on liver fibrosis which may propose the discovery of new antifibrotic agents.

Through ancient eras, medicinal herbs have long been prescribed to prevent and treat liver diseases and have recently gained wider attention due to their availability, long term effectivity and benign side effects (12). Generally, hepatoprotective effects of medicinal herbs is conducted via mechanisms including hindering fibrogenesis, defeating tumorigenesis, eradicating viruses, and suppressing oxidative tissue damage (13). Glycyrrhizae Radix et Rhizoma, also known as licorice root, is commonly consumed to treat viral hepatitis (14). Licorice root main constituents include glycyrrhizc acid (GA), $\beta$-sitosterol, flavonoids, and hydroxycoumarins. GA improves CCL4-induced liver damages by down-regulating proinflammatory mediators (15), as well as its antioxidative action via upregulation of catalase and glutathione-S-transferases (16).

The current study aimed to define the underlying mechanism of anti-fibrotic action of GA on the sub-molecular level by investigating the effects of GA on ITGBL1 binding activity, one of the major controllers of fibrosis, in the $\mathrm{CCl} 4$ rat model of liver cirrhosis.

\section{Materials and methods}

\section{Animals and ethics statement}

This research was approved by the Ethics Committee of faculty of veterinary medicine, Kafrelsheikh University.

\section{Experimental design}

Thirty male albino rats weighing 180-200 g were recruited after 7 days for adaptation to the animal house circumstances (12-hour light/dark cycle). Water and food were supplied ad libitum. The rats were arbitrarily distributed to 4 groups: the control group $(\mathrm{n}=8)$, the $\mathrm{CCl}_{4}$ group $(\mathrm{n}=8)$, the $\mathrm{CCl}_{4}+\mathrm{GA}(\mathrm{n}=8)$ and the GA $150 \mathrm{mg} / \mathrm{kg}$ BW group $(\mathrm{n}=8)$. The liver fibrosis was induced by intraperitoneal (i.p.) injection with $\mathrm{CCl}_{4}$ mixed with olive oil as vehicle in $1: 1$ ratio $(0.2 \mathrm{~mL} / 100 \mathrm{~g} \mathrm{BW})$ twice weekly for 2 weeks followed by i.p. injection of reduced dose $(0.1 \mathrm{~mL} / 100 \mathrm{~g} \mathrm{BW})$ twice weekly for 6 weeks as described by Constandinou (17). GA was given by oral gavage once daily for 8 weeks.

Three days after the last $\mathrm{CCl}_{4}$ injection, rats were sacrificed, and blood samples were taken in the plain tubes and EDTA tubes. Samples in plain tubes were left to clot then centrifuged at $3,000 \mathrm{~g}, 4^{\circ} \mathrm{C}$ for $15 \mathrm{~min}$, to separate serum. The serum samples were stored at $-20^{\circ} \mathrm{C}$ until analyzed. The liver was immediately excised from 
each animal, washed by saline and divided to 2 parts: one part was prepared for histopathological examination, while the other part was preserved frozen at $-80^{\circ} \mathrm{C}$ for both oxidative stress and genetic analysis.

\section{Biochemical analysis}

Activities of liver enzymes ALT, AST, GGT, levels of total protein, albumin and total bilirubin in plasma were measured using commercial kits (Spinreact, Spain) according to the manufacturer's directions. Hepatic SOD, CT and MDA were determined in the hepatic tissue homogenate using (Biodiagnostic, Egypt) kits following the manufacturer's guideline and as previously described $(18,19)$.

\section{RNA extraction and real time PCR}

RNA was extracted out by using Trizol (Invitrogen Co., Carlsbad, CA, USA) and real time PCR performed following the manufacturer's instructions using M-MLV reverse transcriptase (Takara Shuzo Co., Ltd, Japan) and real time PCR Master Mix (SYBR Green) Kit (Toyobo Co., Ltd. Japan). The sequences of ITGBL1 primers were forward 5'TTTGTGAGAAAGGATGGTTTGGT3' and reverse 5'TGCTTTGTTCTTCGGTCATATTA CA3'. GAPDH was used as an internal control. The PCR conditions were $95^{\circ} \mathrm{C}$ for $10 \mathrm{~min}$, and then 40 cycles of $95^{\circ} \mathrm{C}$ for $20 \mathrm{~s}, 54^{\circ} \mathrm{C}$ for $30 \mathrm{~s}$ and $72^{\circ} \mathrm{C}$ for 30 s. Each experiment was carried out thrice in triplicate. The fold-change in mRNA of target gene relative to that of GAPDH was calculated according to previously described (20).

\section{Histopathological examination}

Sections of liver tissue $3 \mu \mathrm{m}$ thickness were obtained from each animal under investigation and fixed in $10 \%$ neutral buffered formalin, then dehydrated in ascending concentration of ethyl alcohol (70: 100\%) followed by staining according to standard protocol of Hematoxylin an Eosin stain (H\&E) as described by Bancroft e al., (21)

\section{Statistical Analysis}

Statistical analysis was carried out using Graphpad prismV5 software package. Results were obtained as means \pm standard deviations
(SD). Statistical analysis was performed using one way analysis of variance (ANOVA) comparisons. Values showing $p<0.05$ was considered as statistically significant.

\section{Results}

\section{Biochemical parameters}

Serum ALT, AST, and GGT activities were determined as indicators of liver damage. As displayed in table 1, significant high levels in the activities of these marker enzymes were recorded in $\mathrm{CCl}_{4}$-intoxicated rats. In contrast, $\mathrm{GA}$ supplement significantly reversed those enzyme activities.

\section{Histopathological Findings}

The liver of control animal (Fig.1A) showed normal hexagonal shape lobule with centrally located blood vessels (central vein) while the peripheral area revealed normal portal areas which contained hepatic artery, portal vein and bile duct. Hepatocytes (large round to polygonal cell with eosinophilic cytoplasm and vesicular nucleus) mostly arranged in radiating manner around the central vein. Similarly, animal treated with GA were with normal limits (Fig.1B). Animals treated with $\mathrm{CCl}_{4}$ (Fig.1C) showed remarkable hepatic distortion associated with centro-lobular hepatic vacuolation and necrosis. Obvious periportal hepatic fibrosis accompanied with noticeable periportal, interlobular and intralobular proliferating fibrous connective tissue (arrowhead) that given the nodular appearance of the hepatic tissue. While diseased animal treated with GA showed distinct decrease of hepatic necrosis and fibrosis (Fig.1D).

\section{Effect of GA treatment on ITGBI expres- sion}

The obtained qPCR results revealed a significant $(\mathrm{P} \leq 0.05)$ upregulation of the fibrosis marker ITGBl gene in liver of $\mathrm{CCl}_{4}$-intoxicated rats as compared to the control group (Table 1). This elevated expression was significantly downregulated following treatment by GA, but still higher than that in the control group. Additionally, no significant difference was noticed between the two control groups (G1, G2). 
Table 1: Effect of GA on biochemical parameters.

\begin{tabular}{|c|c|c|c|c|}
\hline & Normal & GA & $\mathrm{CCl}_{4}$ & $\mathrm{GA}+\mathrm{CCl}_{4}$ \\
\hline AST (IUVL) & $62.8 \pm 3.58^{c}$ & $64.63 \pm 3.18^{c}$ & $346.16 \pm 11.15^{\mathrm{a}}$ & $222.20 \pm 8.93^{b}$ \\
\hline ALT (IUlL) & $59.7 \pm 1.82^{\mathrm{c}}$ & $63.10 \pm 3.95^{c}$ & $253.23 \pm 22.65^{\mathrm{a}}$ & $118.31 \pm 7.56^{b}$ \\
\hline GGT( IU \L) & $67.5 \pm 1.77^{c}$ & $67.44 \pm 3.96^{c}$ & $114.26 \pm 4.54^{\mathrm{a}}$ & $90.18 \pm 3.26^{b}$ \\
\hline Total protein (g\dl ) & $6.54 \pm 0.12^{\mathrm{a}}$ & $6.01 \pm 0.16^{\mathrm{a}}$ & $3.03 \pm 0.24^{\mathrm{c}}$ & $4.41 \pm 0.17^{\mathrm{b}}$ \\
\hline Albumin (g\dl ) & $4.27 \pm 0.20^{\mathrm{a}}$ & $4.02 \pm 0.26^{\mathrm{a}}$ & $2.20 \pm 0.20^{c}$ & $2.92 \pm 0.10^{b}$ \\
\hline Total bilirubin (mg\dl ) & $0.91 \pm 0.06^{\mathrm{c}}$ & $0.90 \pm 0.06^{\mathrm{c}}$ & $1.56 \pm 0.05^{\mathrm{a}}$ & $1.15 \pm 0.04^{\mathrm{b}}$ \\
\hline SOD & $13.03 \pm 0.57^{\mathrm{a}}$ & $13.16 \pm 0.52^{\mathrm{a}}$ & $4.56 \pm 0.46^{\mathrm{c}}$ & $7.19 \pm 0.33^{b}$ \\
\hline Cata & $1.94^{\mathrm{a}}$ & $39.78=$ & $20.25 \pm$ & $27.14 \pm 0.93^{b}$ \\
\hline MDA (nmol $\backslash g$ tissue) & $4.00 \pm 0.36^{c}$ & $4.40 \pm 0.49^{c}$ & $10.51 \pm 0.63^{\mathrm{a}}$ & $7.84 \pm 0.56^{b}$ \\
\hline ITGB1 gene (fold change) & $1.00 \pm 0.09^{\mathrm{c}}$ & $1.51 \pm 0.24^{\mathrm{c}}$ & $11.16 \pm 0.43^{\mathrm{a}}$ & $5.78 \pm 0.29^{b}$ \\
\hline
\end{tabular}

Data were presented as mean \pm SEM. Means carrying different superscript letters are significantly different at $\mathrm{P} \leq 0.05$.
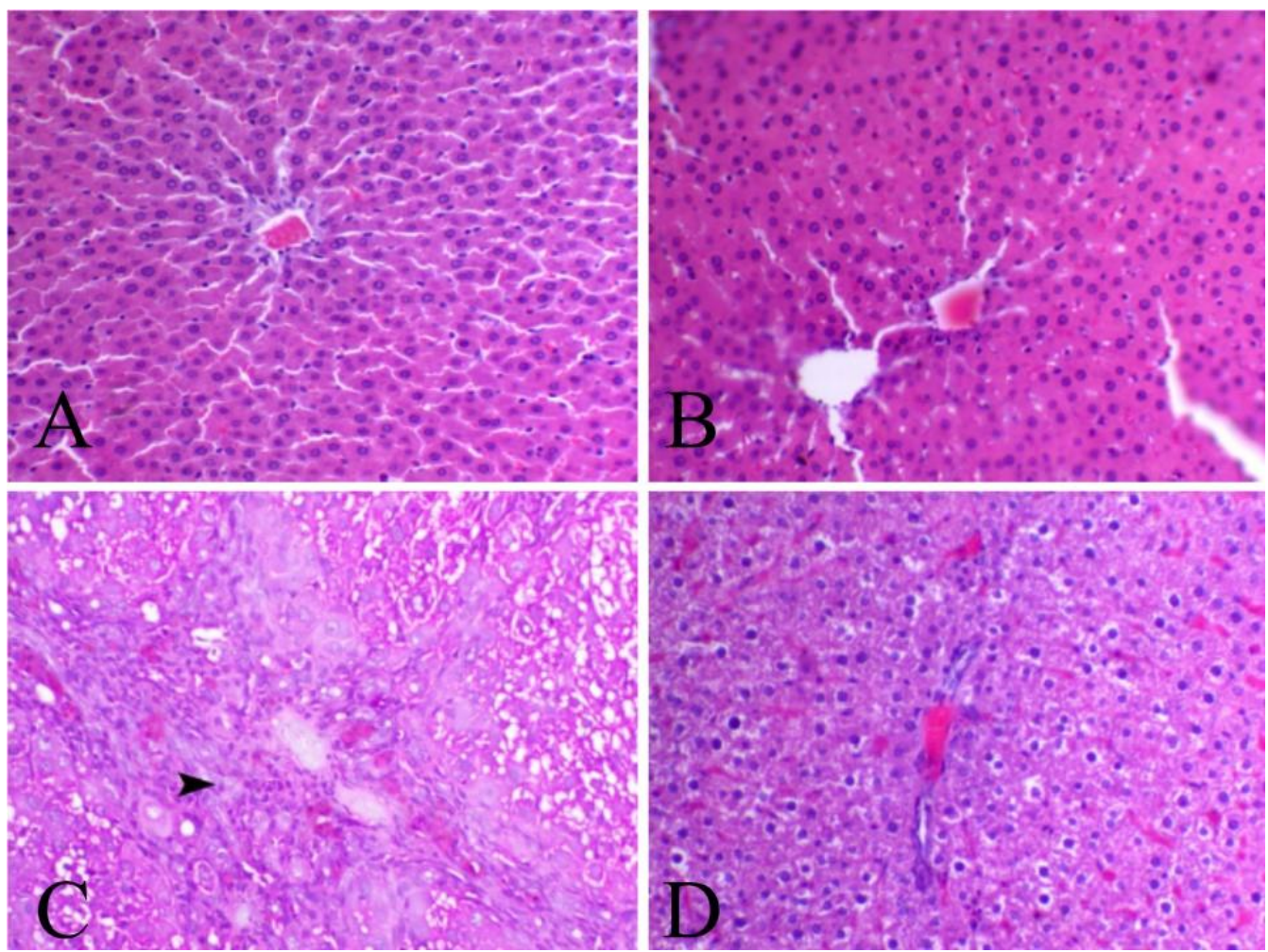

Figure 1: Liver sections of control (A), GA (B), CCL4 (C), and GA+CCL4 (D) groups. H\&E, X200

\section{Discussion}

Inflammation is considered the native defense system of the body against harmful factors with playing a vital role in healing the potential injury. Consistently, liver is highly related to inflammation being highly vascular and vulnerable to serious hepatic injurious factors including toxin metabolites, viruses, fat rich diet and excessive alcohol intake. Prolonged exposure to those agents leads to chronic hepatitis accompanied by fibrosis and subsequent cirrhosis with loss of liver function (22). Accordingly, liver fibrosis in the current study was induced by repeated doses of $\mathrm{CCl}_{4}$ which is the most popular procedure between the liver research laboratories. However, it differs from one laboratory to another in terms of $\mathrm{CCl}_{4}$ dose, route of administration, treatment duration and the expected changes to be studied. 
Communally, $\mathrm{CCl} 4$ mediated liver fibrosis proceeds with elevated serum AST and triglycerides along with liver atrophy (23). Furthermore, these alterations are accompanied by a significant low value of serum albumin indicating advanced loss of hepatic function during extended fibrogenesis. These observations appear consistent with our recorded albumin levels in $\mathrm{CCl} 4$ intoxicated group supported by histopathological lesions.

Cytochrome P450 superfamily of monooxygenases process $\mathrm{CCl} 4$ to the trichloromethyl radical $\left(\mathrm{CCl}_{3}\right)(24)$. Subsequently, this radical damages the key cellular metabolic pathways resulting in altered lipid metabolism (fatty degeneration and steatosis) and decreased protein quantities. Moreover, $\mathrm{CCl}_{3}$ interacts with hepatocytic DNA leading to mutations and the development of HCC. Further oxygenation of $\mathrm{CCl}_{3}$ results in the formation of trichloromethylperoxy radicals $\left(\mathrm{CCl}_{3} \mathrm{OO} *\right)$ initiating lipid peroxidation by breakdown of polyunsaturated fatty acids with reduction of membrane permeability of the plasma membrane extended to mitochondrial and endoplasmic reticulum membrane ended by cellular death. The cellular death eventually develops as zonal or focal necrosis with destruction of normal hepatic tissue construction (22). Biochemically, this severe OS leads to exhaustion of antioxidant activity of SOD and release of high levels of MDA in agreement with our results of the same group. Inappropriately, Fibrosis develops as a healing course in response to inflammation and OS (25) , and can finally progress into HCC (26). Prolonged stimuli of liver injury leads to failure of the regenerative response and substitution of hepatocytes with massive ECM (27) formed mainly by Hepatic stellate cells (HSC) (28).

Interestingly, integrins direct the development of fibrosis regulating inflammation, and by transforming hepatocytes injury into stimulus of matrix-producing mesenchymal cells [HSC/myofibroblasts (MFB)]. The expression of integrins by wide range of cells engaged in liver fibrosis course, as well as their ability to interact with growth factors and other signaling molecules render the concept of targeting integrins an interesting tactic for antifibrotic therapy.
There is no typical treatment plan for hepatic fibrosis, however prophylaxis against liver injury, including minimizing of fat consumption and toxin exposure or administration of an efficient viral hepatitis treatment can resist fibrosis. Surprisingly, no efficient anti-fibrotic drugs have yet to be developed although substantial progress has been made in exploring the pathogenesis of hepatic fibrosis over the last two decades. Medicinal herbs and their bioactive ingredients and extracts could prevent liver fibrosis by two means: through suppression of HSC activity and via inhibition of ECM expansion. HSCs activation are initiated when gene expression and phenotype changes render the inactive cells responsive to other cytokines and stimuli (29).

Oral traditional Chinese herbal medicine has long been used as a non-invasive therapy. The therapeutic mechanisms of herbal medicines and their active compounds have been gradually uncovered and interpreted through in vivo studies. Recent studies have provided a greater understanding of the molecular mechanisms and new therapeutic approaches for liver fibrosis, but it still requires an efficacious remedy. We believe that herbal medicines are sufficiently worthy as potential therapy agents for liver fibrosis if more profound studies about the underlying mechanisms of herbal medicines with improved methodological quality are undertaken.

GA can prevent $\mathrm{CCl}_{4}$ - related liver fibrosis effectively. This is evidenced in restoring the nearly normal hepatic lobule in $\mathrm{GA}+\mathrm{CCl}_{4}$ animals. It could happen by less HSC proliferation, thus reduced levels of collagen, hyaluronic acid (HA), and laminin (LN). In an animal model, GA reduced the death rate of acetaminophen intoxicated mice via inhibition of acetaminopheninduced hepatotoxicity, and reduced the number and area of GGT positive foci, thus reserving liver function and preventing HCC from development (30). GA showed a highly effective chemopreventive action agent against lead acetate hepatic induced oxidative stress in rats because it chelates lead (31), which support the current data obtained from improved oxidative stress markers in the sera of the same group. In 
the molecular level, GA supplement accompanied by suppression of the profibrotic gene ITGBL1, which has a crucial role in management of ECM and the activity of HSC. Up to the published data, it is the first time uncover the role of GA in correlation to integrins during its anti-fibrotic effect. However in concanavalin A- (ConA-) mediated mouse model, GA attenuated ConA-induced hepatitis and fibrosis pgrowth in livers via supression of CD4+ T cell proliferation in response to ConA via the Jun Nterminal kinase (JNK), extracellular signal-regulated kinase (ERK), and phosphoinositide 3kinase (PI3K)/AKT pathways(32).

\section{Conclusion}

Wide attention should be given to integrins as they are infirmly connected to the development of liver fibrosis. Integrins targeting therefore represents an interesting concept of therapeutic strategy, particularly because experimental data recommend potent efficiency of such trend. However, more investigations are needed to identify potentials to specifically antagonize integrins (including specific integrin antagonists, or small nonpeptidic molecules) to stop or reverse fibrosis and avoid detrimental effect of such inhibition.

\section{References}

1. Anthony P P, Ishak K G, Nayak N C, Poulsen H E, Scheuer P J, Sobin L H. The morphology of cirrhosis. Recommendations on definition, nomenclature, and classification by a working group sponsored by the World Health Organization, J Clin Pathol 1978; 31(5): 395-414.

2. Buti M, Fung S, Gane E, Afdhal N H, Flisiak R, Gurel S, Flaherty J F, Martins E B, Yee L J, Dinh P, Bornstein J D, Mani Subramanian G, Janssen H L, George J, Marcellin P. Long-term clinical outcomes in cirrhotic chronic hepatitis B patients treated with tenofovir disoproxil fumarate for up to 5 years, Hepatol Int 2015; 9(2): 243-50.

3. Blachier M, Leleu H, Peck-Radosavljevic M, Valla D C, Roudot-Thoraval F. The burden of liver disease in Europe: a review of available epidemiological data, J Hepatol 2013; 58(3): 593608.

4. Disease G B D, Injury I, Prevalence C. Global, regional, and national incidence, prevalence, and years lived with disability for 328 diseases and injuries for 195 countries, 1990-2016: a systematic analysis for the Global Burden of Disease Study 2016, Lancet 2017; 390(10100): 1211-59.

5. Heidelbaugh J J, Bruderly M. Cirrhosis and chronic liver failure: part I. Diagnosis and evaluation, Am Fam Physician 2006; 74(5): 756-62.

6. Hernandez-Gea V, Friedman $\mathrm{S}$ L. Pathogenesis of liver fibrosis, Annu Rev Pathol 2011; 6: 425-56.

7. Pellicoro A, Ramachandran $\mathrm{P}$, Iredale $\mathrm{J} \mathrm{P}$, Fallowfield J A. Liver fibrosis and repair: immune regulation of wound healing in a solid organ, Nat Rev Immunol 2014; 14(3): 181-94.

8. Luo B H, Springer T A. Integrin structures and conformational signaling, Curr Opin Cell Biol 2006; 18(5): 579-86.

9. Conroy K P, Kitto L J, Henderson N C. alphav integrins: key regulators of tissue fibrosis, Cell Tissue Res 2016; 365(3): 511-9.

10. Patsenker E, Popov Y, Stickel F, Jonczyk A, Goodman S L, Schuppan D. Inhibition of integrin alphavbeta6 on cholangiocytes blocks transforming growth factor-beta activation and retards biliary fibrosis progression, Gastroenterology 2008; 135(2): 660-70.

11. Wang M, Gong Q, Zhang J, Chen L, Zhang Z, Lu L, Yu D, Han Y, Zhang D, Chen P, Zhang X, Yuan Z, Huang J, Zhang X. Characterization of gene expression profiles in $\mathrm{HBV}$-related liver fibrosis patients and identification of ITGBL1 as a key regulator of fibrogenesis, Sci Rep 2017; 7: 43446.

12. Elgazar A A, Selim N M, Abdel-Hamid N M, El-Magd M A, El Hefnawy H M. Isolates from Alpinia officinarum Hance attenuate LPS induced inflammation in HepG2: Evidence from In Silico and In Vitro Studies, Phytotherapy Research 2018; 32(7): 1273-88.

13. Del Prete A, Scalera A, Iadevaia M D, Miranda A, Zulli C, Gaeta L, Tuccillo C, Federico A, Loguercio C. Herbal products: benefits, limits, and applications in chronic liver disease, Evid Based Complement Alternat Med 2012; 2012: 837939.

14. Hanje A J, Fortune B, Song M, Hill D, McClain C. The use of selected nutrition supplements and complementary and alternative medicine in liver disease, Nutr Clin Pract 2006; 21(3): 255-72.

15. Feng C, Wang H, Yao C, Zhang J, Tian Z. Diammonium glycyrrhizinate, a component of traditional Chinese medicine Gan-Cao, prevents murine T-cell-mediated fulminant hepatitis in IL- 
10- and IL-6-dependent manners, Int Immunopharmacol 2007; 7(10): 1292-8.

16. van Rossum T G, Vulto A G, de Man R A, Brouwer J T, Schalm S W. Review article: glycyrrhizin as a potential treatment for chronic hepatitis C, Aliment Pharmacol Ther 1998; 12(3): 199-205.

17. Constandinou C, Henderson N, Iredale J P. Modeling liver fibrosis in rodents, Methods Mol Med 2005; 117: 237-50.

18. Alzahrani F A, El-Magd M A, AbdelfattahHassan A, Saleh A A, Saadeldin I M, El-Shetry E S, Badawy A A, Alkarim S. Potential Effect of Exosomes Derived from Cancer Stem Cells and MSCs on Progression of DEN-Induced HCC in Rats, Stem Cells International 2018; 2018: 17 https://doi.org/10.1155/2018/8058979.

19. El-Bayomi K M, Saleh A A, Awad A, ElTarabany M S, El-Qaliouby H S, Afifi M, El-Komy S, Essawi W M, Almadaly E A, El-Magd M A. Association of CYP19A1 gene polymorphisms with anoestrus in water buffaloes, Reproduction, Fertility and Development 2018; 30(3): 487-497.

20. Abd-Allah S H, Shalaby S M, Abd-Elbary E, Saleh A A, El-Magd M A. Human peripheral blood CD34+ cells attenuate oleic acid-induced acute lung injury in rats, Cytotherapy 2015; 17(4): 443-53.

21. Bancroft J D, Stevens A, Turner D R, Theory and practice of histological techniques, Fourth Edition ed., Churchill Livingstone, New York, 1996.

22. Chatterjee R, Mitra A. An overview of effective therapies and recent advances in biomarkers for chronic liver diseases and associated liver cancer, Int Immunopharmacol 2015; 24(2): 335-345.

23. Paquet K J, Kamphausen U. The carbontetrachloride-hepatotoxicity as a model of liver damage. First report: Long-time biochemical changes, Acta Hepatogastroenterol (Stuttg) 1975; 22(2): 84-8.
24. Li X X, Zheng Q C, Wang Y, Zhang H X. Theoretical insights into the reductive metabolism of $\mathrm{CCl} 4$ by cytochrome P450 enzymes and the CCl4-dependent suicidal inactivation of P450, Dalton Trans 2014; 43(39): 14833-40.

25. Chen P J, Huang C, Meng X M, Li J. Epigenetic modifications by histone deacetylases: Biological implications and therapeutic potential in liver fibrosis, Biochimie 2015; 116: 61-9.

26. Santos J C, Valentim I B, de Araujo O R, Ataide Tda R, Goulart M O. Development of nonalcoholic hepatopathy: contributions of oxidative stress and advanced glycation end products, Int J Mol Sci 2013; 14(10): 19846-66.

27. Elpek G O. Cellular and molecular mechanisms in the pathogenesis of liver fibrosis: An update, World J Gastroenterol 2014; 20(23): 726076.

28. Reeves H L, Friedman S L. Activation of hepatic stellate cells--a key issue in liver fibrosis, Front Biosci 2002; 7: d808-26.

29. Friedman S L. Mechanisms of hepatic fibrogenesis, Gastroenterology 2008; 134(6): 165569.

30. Wan X Y, Luo M, Li X D, He P. Hepatoprotective and anti-hepatocarcinogenic effects of glycyrrhizin and matrine, Chem Biol Interact 2009; 181(1): 15-9.

31. Rahman S, Sultana S. Chemopreventive activity of glycyrrhizin on lead acetate mediated hepatic oxidative stress and its hyperproliferative activity in Wistar rats, Chem Biol Interact 2006; 160(1): 61-9.

32. Tu C T, Li J, Wang F P, Li L, Wang J Y, Jiang W. Glycyrrhizin regulates CD4+T cell response during liver fibrogenesis via JNK, ERK and PI3K/AKT pathway, Int Immuno-pharmacol 2012; 14(4): 410-21. 